\title{
MAN.I 7 - Vaccines clinical research model at Basic Health Units in Rio de Janeiro
}

Maria Letícia Borges dos Santos ${ }^{1}$; Maria de Lourdes de Sousa Maia ${ }^{1 *}$; Claudia Ferreira Motta ${ }^{1}$; Jaqueline Toledo de Oliveira Figueira ${ }^{1}$; Daniele Fernandes de Aguiarr ${ }^{1}$; Dayana Cristina Vieira de Souza ${ }^{1}$; Celia Menezes Cruz Marques ${ }^{2}$.

1Fiocruz/Bio-Manguinhos;

2Secretaria Municipal de Saúde do Rio de Janeiro.

Introduction: The Ministries of Health (MS) and Science and Technology (MCTIC) launched a public notice in 2005 for the development of all clinical trial phases for pharmaceuticals, procedures, equipment and diagnostic kits. Nineteen Centers were selected, composing the National Clinical Research Network for Academic Hospitals (RNPC). However, we understood that the sites most likely to find healthy subjects for vaccines studies are not part of this network, as they don't belong to any academic institution or hospital. The more appropriate sites for vaccine studies are the Basic Health Units (BHU) from the Municipality Secretary of Health of Rio de Janeiro (SMSRJ).

Objective: Analyze the method established with SMSRJ for vaccine clinical trials accomplishment in BHUs, with the development of a structured network of permanent and temporary Research Centers and qualified professionals, to validate financial and execution viability.

Methodology: Based on clinical protocol and target population for each study, several activities are performed jointly by ASCLIN and SMSRJ. After the definition of the principal investigator, a situational and structural survey is made for BHUs, considering: political situation, population coverage and demand, proximity with samples processing laboratory, conditions for investigational products storage and adequate infrastructure. Based on this survey BHUs are selected and starts the submission to IRBs. After approval, physical infrastructure improvements are made with acquisition of equipment, files and materials, training is provided on Good Clinical Practices and study documentation for BHUs teams, and study dissemination occurs on District Councils, Public Health Agents meeting and Neighborhood Associations. Follow-up and actions for risk mitigation are executed during the study, which take up to 2,5 years, and at the end is performed study close-out with workshops for results disclosure.

Results: It was identified that this methodology allowed execution of vaccine studies with less costs related to infrastructure and execution agility due to proximity relations with communities. Additionally, it enabled clinical trial qualification for more than 100 professionals and adaptation of more than 16 BHU into Research Centers, with permanent structuring of two Centers, one of them with a biological samples processing laboratory of Level 2 Biosafety (NB2). Finally it provided reproducibility as other studies were accomplished all around Brazil using similar approach with local institutions.

Conclusion: The partnership model implemented allowed SMSRJ and other institutions to train professionals on the binomial Health-Disease, beyond the BHU traditional approach. The local infrastructure improvements and the incorporation of equipment in each BHU strengthened the partnership. With each study executed Bio-Manguinhos managed to increase the level of awareness and importance of vaccination within the local population. This methodology allowed the achievement of studies with cost value instead of market value, which benefits all, specially the Brazilian Public Health with the study results being applied in SUS.

Keywords: BHU; Clinical Research; Research Centres 